\title{
Technology adoption under embodiment: A two-stage optimal control approach*
}

\author{
Raouf Boucekkine ${ }^{\dagger}$ Cagri Saglam ${ }^{\ddagger}$ Thomas Vallée ${ }^{\S}$ \\ to be published in Macroeconomic Dynamics
}

\begin{abstract}
We use two stage optimal control techniques to solve some adoption problems under embodied technical change. We first solve a benchmark problem without learning behavior. At the date of switching, the consumption level is shown to drop, as the relative price of capital goes down (obsolescence). In such a case, the economy sticks to the initial technology, or immediately switches to a new technology with a higher level of embodiment, depending on how the obsolescence costs compare to the induced growth advantage. In a second step, we introduce learning. The learning curve involves fixed costs and incentives to wait as well. Adoption is shown to depend on the growth advantage of switching net of obsolescence and learning fixed costs. The economy will switch if and only if this indicator is positive. If it is big enough to "compensate" the option of waiting, then the economy switches immediately. Otherwise, the economy waits.
\end{abstract}

Keywords: Optimal control, adoption, learning, embodiment Journal of Economic Literature: E22, E32, O40, C63.

*The authors warmly thank two anonymous referees for their crucial observations and suggestions. R.Boucekkine and C. Saglam acknowledge the support of the Belgian research programme "Action de Recherches Concertée" (99/04-235), and of the Belgian Federal Government (PAI P5/10).

†IRES and CORE, Université catholique de Louvain, Louvain-la-Neuve (Belgium). Email: boucekkine@ires.ucl.ac.be.

$\ddagger$ IRES, Université catholique de Louvain. E-mail: saglam@ires.ucl.ac.be

$\S$ Université de Nantes. E-mail: Thomas.Vallee@sc-eco.univ-nantes.fr 


\section{Introduction}

Technology adoption has become one of the principal research topics in growth theory in the recent years. The considerable weight of adoption costs (twenty times higher than those for innovation, see for example Jovanovic, 1997) has directed interest in the profession towards the study of problems of diffusion and adoption of new technologies. The recent boom of the information technologies and the associated employment and growth opportunities has made the latter study even more urgent from the economic policy point of view. ${ }^{1}$

Technology adoption is costly in that it requires some specific vintage physical and human capital (see the survey of Greenwood and Jovanovic, 1998) and involves learning costs. Indeed technological upgrading implies the depreciation of the pre-existing specific human capital (Parente, 1994) and a slow learning process during which the economy is unable to run the new technology at its best productivity level. In order to avoid this phase, an economy would need to gather engineers and scientists with some specific skills, which is hardly feasible, so that technology adoption could even lead to a productivity slowdown (Greenwood and Yorukoglu, 1997). A slightly different mechanism is put forward by Galor and Tsiddon (1997) (see also Galor and Moav, 2000). A technological acceleration induces an erosion effect on the level of human capital of each individual. Though the acceleration does allow the individuals to operate at a higher productivity (the so-called productivity effect), the erosion effect is predominant in the short run, giving rise to a productivity slowdown. The latter considerations are specially true if technological progress is embodied in the new capital goods, as the technological advances conveyed by the information technologies are. Since embodiment is both qualitatively and quantitatively one of the most relevant features of growth specially since the seventies (see Greenwood, Hercowitz and Krusell, 1997), the adoption problem is increasingly crucial.

A comprehensive (but yet incomplete) appraisal of the technology problem would treat the two following questions: Given the initial state of the economy, including its technological and skill states, is it optimal to switch to another more productive but capital and skill specific technology? If the decision is to adopt, what is the optimal timing of adoption? The first question is the basic one, but the second is far from accessory. A too quick and/or massive adoption would be a disaster, on one hand because the return to adoption, just like the return to innovation, is truly uncertain, ${ }^{2}$ and on the other hand, because of the lack of complementarity between the new technology and the actual structure of the economy (for example, the composition of skills in the economy, see Galor and Tsiddon, 1997). In the case 
of the technologies embodied in capital goods, the issue is even more crucial. These technologies are associated with non-negligible obsolescence costs (see Krusell, 1998, and Boucekkine, del Río and Licandro, 2002). As the pace of embodied technological progress accelerates, a large amount of vintage capital goods will be out-graded and withdrawn from the productive sectors. If upgrading technologies induces significant obsolescence costs, it is unclear why the economy should upgrade. If additionally one takes into account the learning costs inherent to adoption, the issue becomes even more challenging. Under which conditions would an economy switch to a more productive technology knowing that switching involves obsolescence and learning costs? What is the optimal timing of switching?

Some early contributions to the optimal adoption problem have stressed its economic and technical complexity. In Parente (1994) and Galor and Moav (2000), the adoption problem results from the depreciation of the pre-existing specific human capital, and the need to learn the new technology. In Jovanovic and Nyarko's model, the learning process is Bayesian in nature and as long as the economic agents do not estimate accurately some productivity parameters, an output loss results. It is a partial equilibrium set-up, and the considered Bayesian structure makes it difficult to think about a general equilibrium extension. In contrast, Parente, and Galor and Moav consider general equilibrium models. The latter use a discrete time two periods overlapping generations framework, and are able to analytically characterize the dynamics of technology adoption. Parente uses a continuous time infinite horizon representative agent setting, and is unable to provide such a characterization. Indeed, the endogeneity of adoption timing in his framework disables any analytical inspection into the dynamics of technology adoption despite the linearity of the production function and other simplifying assumptions. For such a purpose, only a computational approach works (see an extension of Parente's model in Iacompetta, 2001). Moreover and in contrast to Jovanovic and Nyarko's setting, there is no possibility to stick to a given technology, upgrading always occurs, which makes Parente's model unadapted when one has to handle technological sclerosis cases.

Our paper is a technical contribution to the literature of technology adoption allowing to address the problems mentioned just above. We solve technology adoption optimization programs in continuous time. Technological progress is assumed to be embodied in capital goods. In addition to eventual learning costs, our problem involves obsolescence costs. The economy starts with a given technological menu, consisting of the levels of both embodied and disembodied technological progress. As in Greenwood, Hercowitz and Krusell (1997), the embodied technological progress variable does indeed measure the productivity in the capital goods sector, while the disembodied 
or neutral technological progress variable amounts to factor productivity in the consumption goods sector. However, in contrast to these authors, we consider that the production technology in the latter sector is AK, ie. constant returns to scale with respect to capital, so that long term growth is no longer exogenous. We assume that another menu is available from the beginning with a higher a level of embodied technical progress but with a lower level of disembodied technological progress (to reflect the loss of expertise in the new technology after switching). It is shown that a higher level of embodied technical change is associated with a decrease in the relative price of capital, which rises the user cost of capital by the so called obsolescence costs, a well known property since Solow (1960). However, having a more efficient capital sector is likely to trigger growth, and may offset the obsolescence costs. How should the economy deal with this trade-off? Can we characterize an optimal timing for adoption?

In this paper, we study various adoption problems within the framework described just above. In line with the above mentioned literature on technology adoption, we also analyze the role of learning in this process. In contrast to Parente (1994), we just allow for one switch (or a finite number of switches, see Saglam, 2002). From the point of view of technology monitoring policy, this is far from a shortcoming. In very sharp contrast to the existing literature, we are able to derive analytical results using two stage optimal control techniques (Tomiyama, 1985, Tomiyama and Rossana, 1989, and Makris, 2001). The approach uses standard optimal control theory but it is constructed as a dynamic programming method. We will show that these techniques are extremely easy to implement and prove very efficient when one is facing optimal adoption problems. Moreover, the economic insight that one can gain from using the two stage optimal control framework is also appreciable. For example, we will see later on that we can generate the three possible decisions: immediate adoption, and sticking to the initial technology as corner solutions, and delayed adoption (finite delay) as the interior solution. Thus, the set-up can perfectly be used to finely investigate the determinants of adoption delays.

In particular, we will study how learning can affect adoption delays by considering a rich learning curve. Learning involves some fixed costs but it may be partly controllable by the economy. Indeed, the amount to be learnt may depend on the timing of adoption for strategic reasons. For example, there may be an incentive to wait in order to take advantage of the experience of the first movers. There are many other foundations of strategic behavior in such a context (see Greenwood and Jovanovic, 1998), all attempting to reproduce the observed diffusion curves. We will introduce such an ingredient in our model, and study how it interacts with the obsolescence costs and the fixed learning costs induced by technology switching. The optimal adoption 
pace is then finely described in connection with the latter aspects.

To this end, we will structure the paper as follows. Section 2 will solve a preliminary adoption problem with embodied technical change but without learning curves. The problem will be solved both under a finite and infinite optimization time horizon. We will make clear from the beginning of this section how two stage optimal control works. Section three introduces learning curves. Section 4 concludes.

\section{The basic model}

Consider an economy inhabited by a representative agent which intertemporal utility function is

$$
\int_{0}^{T} u(C(t)) e^{-\rho t} d t
$$

where $u($.$) is increasing and strictly concave. We shall consider first that$ the optimization horizon, $T$, is finite in order to illustrate one of the fundamental properties of optimal adoption timing: Its extreme sensitivity to the optimization horizon. $\rho$ is the time discounting parameter. This parameter will be also shown to be decisive in the adoption decision. The production function in the consumption sector is simply AK, and the consumption good is either used for consumption or as an input, $I$, in the production of the capital goods:

$$
Y=C+I=F(K)=A K,
$$

where $A>0$ is the marginal productivity of capital. ${ }^{3}$ The production function in the capital goods sector is, as in Greenwood, Hercowitz and Krusell (1997):

$$
\dot{K}=q I=q[A K-c],
$$

assuming without any loss of generality that the capital depreciation rate is nil. $q$ measures the productivity in this sector, and as such, it represents the embodied technical progress variable. Note that a rise in $q$ will only affect the new capital goods, in contrast to an increase in $A$, which is meant to have the same effect on all capital goods whatever the date of the their production, whatever their vintage. ${ }^{4}$ In this sense, $A$ is neutral and $q$ is investment specific. We finally, assume that $K(0)=K_{0}$ is given but $K(T) \geq 0$ is free.

Hereafter, we shall represent any technical menu by a pair of positive numbers $(A, q)$. We assume that the economy starts with a menu $\left(A_{1}, q_{1}\right)$. However, another menu $\left(A_{2}, q_{2}\right)$ is available from $t=0$ involving: $q_{2}>q_{1}$ but $A_{2}<A_{1}$. Roughly speaking, the economy can switch to a second technical regime with a more efficient capital goods sector but with a less efficient consumption 
goods sector. Implicit in the latter the assumption that the economy masters the first technology but not the second, which implies say a lower expertise in the use of the new capital goods associated with the second technology. This assumption is completely in line with Parente (1994), and even more with Greenwood and Jovanovic (2001). We will omit learning effects in this benchmark case. Assume that at date $t_{1} \in[0, T]$, the economy does operate the switch. Then, the production function of the capital goods (ie. the state equation of the underlying optimal control problem) can be decomposed as follows:

$$
\dot{K}=q_{1}\left[A_{1} K-C\right]
$$

if $0 \leq t<t_{1}$, and

$$
\dot{K}=q_{2}\left[A_{2} K-C\right]
$$

if $t_{1}<t \leq T . t_{1}$ is the switching time. Given the trade-off described above, what would the optimal switching time of an economy maximizing the welfare of a representative agent subject to the state equation above? Under which conditions the economy switches immediately (ie. at $t=0$, delays switching or sticks to the first technology forever? If the economy delays switching, what are the determinants of this optimal delay? We will address precisely these issues and we will undertake the task analytically using two stage optimal control. The next section briefly describes the resolution procedure.

\subsection{The two stage optimal control technique}

Summarizing the discussion just before, our optimal control problem is

$$
\max _{C, t_{1}} \int_{0}^{T} u(C(t)) e^{-\rho t} d t
$$

subject to (1) and (2), $K_{0}$ given and $K(T) \geq 0$ free. Note that we can rewrite our objective function as follows:

$$
U\left(C, t_{1}\right)=\int_{0}^{t_{1}} u(C) e^{-\rho t} d t+\int_{t_{1}}^{T} u(C) e^{-\rho t} d t
$$

As the welfare performances of the two possible technological regimes are additively separable, the use of two stage optimal control is most natural. The approach is carefully described and theoretically founded in Tomiyama (1985) and Tomiyama and Rossana (1989). An extension to infinite horizons programs has been very recently provided by Makris (2001). As mentioned in the introduction, the method proceeds by solving a sequence of conventional 
Pontryagin problems. In our case, the sequence contains two problems, which corresponds to the number of possible technological regimes, but as it will be clear in a moment, the method should work at least for any finite number of problems (see Saglam, 2002). Applied to our model, the method works like this:

1. The new technology problem step: Assume that the economy does switch to the new technology at $t_{1}$. Furthermore, assume also that initial capital stock for the new technology $K\left(t_{1}\right)=K_{1}$ is given. For $t_{1}$ and $K_{1}$ given, maximize $U_{2}\left(K_{1}, t_{1}\right)=\int_{t_{1}}^{T} u(C) e^{-\rho t} d t$, subject to the state equation (2), $\dot{K}=q_{2}\left[A_{2} K-C\right], t_{1}<t \leq T$, with $K(T)$ free. Denote by $\lambda_{2}(t)$ and by $H_{2}\left(K, C, t, \lambda_{2}\right)=-u(C(t)) e^{-\rho t}+\lambda_{2}\left(q_{2} A_{2} K(t)-q_{2} C(t)\right)$, respectively the co-state variable and Hamiltonian associated with this Pontryagin problem. ${ }^{5}$ Assuming that a maximum exists, denote by $U_{2}^{*}\left(K_{1}, t_{1}\right)$ and $H_{2}^{*}\left(K_{1}, t_{1}\right)$, respectively the optimal welfare performance of the new technology regime and the optimal Hamiltonian value for $t_{1}$ and $K_{1}$ given.

2. The old technology problem step: Maximize $U\left(C, t_{1}\right)=\int_{0}^{t_{1}} u(C) e^{-\rho t} d t+$ $U_{2}^{*}\left(K_{1}, t_{1}\right)$, subject to the state equation (1), $\dot{K}=q_{1}\left[A_{1} K-C\right], 0 \leq t<t_{1}$, $K_{0}$ given and $K_{1}=K\left(t_{1}\right)$ free. This is done in two sub-steps:

2.1. Solve the problem for fixed $K_{1}$ and $t_{1}$, with $\lambda_{1}(t)$ and $H_{1}\left(K, C, t, \lambda_{1}\right)=$ $-u(C(t)) e^{-\rho t}+\lambda_{1}\left(q_{1} A_{1} K(t)-q_{1} C(t)\right)$, respectively the corresponding costate variable and Hamiltonian. Denote by $H_{1}^{*}\left(K_{1}, t_{1}\right)$ the resulting optimal Hamiltonian value.

2.2. The matching conditions: Continuity and optimality. Find the optimal values for $K_{1}$ and $t_{1}$. For interior optimal solutions, $K_{1}^{*}$ and $t_{1}^{*}$, to exist, the following equations should hold:

$$
\begin{aligned}
\lambda_{1}\left(t_{1}\right) & =-\frac{\partial U_{2}^{*}\left(K_{1}, t_{1}\right)}{\partial K_{1}} \\
H_{1}^{*}\left(K_{1}, t_{1}\right) & =\frac{\partial U_{2}^{*}\left(K_{1}, t_{1}\right)}{\partial t_{1}}
\end{aligned}
$$

If $U_{2}\left(K_{1}, t_{1}\right)$ is twice continuously differentiable in $K_{1}$ and $t_{1}$, these conditions become:

$$
\begin{aligned}
\lambda_{1}\left(t_{1}^{*}\right) & =\lambda_{2}\left(t_{1}^{*}\right), \\
H_{1}^{*}\left(K_{1}^{*}, t_{1}^{*}\right) & =H_{2}^{*}\left(K_{1}^{*}, t_{1}^{*}\right) .
\end{aligned}
$$

As one can easily see, equation (6) featuring the optimality of the $K_{1}$ 's choice ensures the continuity of the co-state variable at $t_{1}$. Equation (7) is the optimality condition for an interior switching time to exist. 
3. Sufficient condition for maximum:

$$
\frac{\partial H_{2}^{*}\left(K_{1}, t_{1}\right)}{\partial t_{1}}-\frac{\partial H_{1}^{*}\left(K_{1}, t_{1}\right)}{\partial t_{1}}<0 .
$$

4.Corner solutions. Assume that $U_{2}\left(K_{1}, t_{1}\right)$ is twice continuously differentiable in $K_{1}$ and $t_{1}$, which turns out to be the case in all our applications. Consider the case where the equations (6)-(7) have no solution in $t_{1}$ such that $0<t_{1}<T$. Two corner solutions are still possible.

(i) Immediate switching: $t_{1}^{*}=0$ if $H_{1}^{*}\left(K_{0}, 0\right) \geq H_{2}^{*}\left(K_{0}, 0\right)$.

(ii) Technological sclerosis: The economy will never switch in the time interval $[0 T]$ if and only if $H_{1}^{*}\left(K_{1}^{*}, t_{1}^{*}\right)<H_{2}^{*}\left(K_{1}^{*}, t_{1}^{*}\right)$ for every $t_{1}$ in $[0 T[$ and $H_{1}^{*}\left(K^{*}(T), T\right) \leq H_{2}^{*}\left(K^{*}(T), T\right) .{ }^{6}$

We shall apply this method to our basic model and give the details of the corresponding computations in the next section. The algebraic details will be omitted in further applications of the method.

\subsection{Solving the model}

We will follow closely the method described above starting by the solution of the Pontryagin problem corresponding to the new technological regime. We take the logarithmic utility case to ease the exposition, the method still produces closed-form solutions for any other isoelastic utility function for example. Concise economic interpretations will follow the technical exposition.

1. Our optimal control problem is:

$$
\max _{C} U_{2}\left(C, t_{1}\right)=\int_{t_{1}}^{T} \ln (C(t)) e^{-\rho t} d t
$$

subject to $\dot{K}=q_{2} A_{2} K(t)-q_{2} C(t)$, with $K_{1}$ given and $K(T) \geq 0$ free. With the corresponding Hamiltonian defined as: $H_{2}\left(K, C, t, \lambda_{2}\right)=-u(C(t)) e^{-\rho t}+$ $\lambda_{2}\left(q_{2} A_{2} K(t)-q_{2} C(t)\right)$, the first order necessary conditions are standard:

$$
\begin{aligned}
& \dot{K}^{*}=\left[\partial H_{2} / \partial \lambda_{2}\left(K^{*}, \lambda_{2}^{*}, C^{*}, t\right)\right], \\
& \dot{\lambda}_{2}^{*}=-\left[\partial H_{2} / \partial K\left(K^{*}, \lambda_{2}^{*}, C^{*}, t\right)\right], \\
& \lambda_{2}^{*}(T) \leq 0 \text { and } K^{*}(T) \lambda_{2}^{*}(T)=0
\end{aligned}
$$

and with

$$
\min _{C} H_{2}\left(K^{*}(t), \lambda_{2}^{*}(t), C, t\right)=H_{2}\left(K^{*}(t), \lambda_{2}^{*}(t), C^{*}(t), t\right) \text { a.e. on }\left[t_{1}, T\right]
$$


Since $C^{*}$ clearly depends on the choice of $t_{1}$ and $K_{1}$ it is obvious that : $U_{2}^{*}=U_{2}^{*}\left(K_{1}, t_{1}\right)$. The (trivial) integration of these necessary conditions gives us the following results ${ }^{7}$ :

$$
\begin{aligned}
K(t) & =e^{\left(q_{2} A_{2}\right) t}\left[e^{-\left(q_{2} A_{2}\right) t_{1}} K_{1}+\frac{q_{2} a_{0}}{\rho}\left(e^{-\rho t}-e^{-\rho t_{1}}\right)\right], \\
C(t) & =a_{0} e^{\left(q_{2} A_{2}-\rho\right) t} \\
\lambda_{2}(t) & =-\frac{1}{q_{2} a_{0}} e^{-\left(q_{2} A_{2}\right) t} .
\end{aligned}
$$

Given the terminal point condition (11), one may find that

$$
a_{0}=-\frac{\rho K_{1}}{q_{2}} \frac{e^{-\left(q_{2} A_{2}\right) t_{1}}}{\left(e^{-\rho T}-e^{-\rho t_{1}}\right)}
$$

And thus, at $t_{1}$, we have

$$
\begin{aligned}
C\left(t_{1}\right) & =\frac{\rho K_{1}}{q_{2}} \frac{e^{-\rho t_{1}}}{\left(e^{-\rho t_{1}}-e^{-\rho T}\right)}, \\
\lambda_{2}\left(t_{1}\right) & =\frac{\left(e^{-\rho T}-e^{\rho t_{1}}\right)}{\rho K_{1}} .
\end{aligned}
$$

This finally allows us to compute the optimal welfare performance of the new technology regime in terms of $t_{1}$ and $K_{1}$.

$$
U_{2}^{*}\left(K_{1}, t_{1}\right)=\int_{t_{1}}^{T} \ln \left[-\frac{\rho K_{1}}{q_{2}} \frac{e^{-\rho t_{1}} e^{\left(q_{2} A_{2}-\rho\right) t}}{\left(e^{-\rho T}-e^{-\rho t_{1}}\right)}\right] e^{-\rho t} d t .
$$

Notice that $U_{2}^{*}\left(K_{1}, t_{1}\right)$ is trivially twice differentiable with respect to both $K_{1}$ and $t_{1}$. Accordingly one can check that the following relations hold:

$$
\begin{gathered}
\frac{\partial U_{2}^{*}\left(K_{1}, t_{1}\right)}{\partial K_{1}}=-\lambda_{2}\left(t_{1}\right), \\
\frac{\partial U_{2}^{*}\left(K_{1}, t_{1}\right)}{\partial t_{1}} \equiv H_{2}^{*}\left(K_{1}, t_{1}\right) .
\end{gathered}
$$

2. Now we have to solve the following Pontryagin problem

$$
\max _{\left\{C, t_{1}\right\}} U_{1}(C, t)=\int_{0}^{t_{1}} \ln (C(t)) e^{-\rho t} d t+U_{2}^{*}\left(K_{1}, t_{1}\right)
$$

subject to $\dot{K}(t)=q_{1} A_{1} K(t)-q_{1} C(t), 0 \leq t<t_{1}$, with $K_{0}$ given and $K_{1}=K\left(t_{1}\right)$ free. Assuming that $t_{1}^{*}$ is an interior point in $[0, T]$ makes the 
constraint $0 \leq t_{1} \leq T$ inactive and we are left with an auxiliary problem for the old technology with free end point and free terminal time.

2.1 For fixed $t_{1}$ and $K_{1}$, the Pontryagin maximum principles allow to write straightforwardly the first order conditions as in step 1. Integrating them gives:

$$
\begin{aligned}
K(t) & =e^{\left(q_{1} A_{1}\right) t}\left[K_{0}+\frac{q_{1} \bar{a}_{0}}{\rho}\left(e^{-\rho t}-1\right)\right], \\
C(t) & =\bar{a}_{0} e^{\left(q_{1} A_{1}-\rho\right) t} \\
\lambda_{1}(t) & =-\frac{1}{q_{1} \bar{a}_{0}} e^{-\left(q_{1} A_{1}\right) t} .
\end{aligned}
$$

2.2. In order to determine optimal $K_{1}$ and $t_{1}$, we use the continuity and optimality conditions (6) and (7). By means of (6), we can find that :

$$
\bar{a}_{0}=-\frac{\rho K_{1}}{q_{1}} \frac{e^{-\left(q_{1} A_{1}\right) t_{1}}}{\left(e^{-\rho T}-e^{-\rho t_{1}}\right)} .
$$

And thus, at $t_{1}$, we have

$$
\begin{aligned}
C\left(t_{1}\right) & =\frac{\rho K_{1}}{q_{1}} \frac{e^{-\rho t_{1}}}{\left(e^{-\rho t_{1}}-e^{-\rho T}\right)}, \\
\lambda_{1}\left(t_{1}\right) & =\frac{\left(e^{-\rho T}-e^{-\rho t_{1}}\right)}{\rho K_{1}}, \\
K\left(t_{1}\right) & =\frac{\left(e^{\rho\left(T-t_{1}\right)}-1\right)}{\left(e^{\rho T}-1\right)} e^{\left(q_{1} A_{1}\right) t_{1}} K_{0} .
\end{aligned}
$$

Now utilizing the optimality condition (7), we can determine the (interior) optimal time of switching to the new technology as follows:

$$
\begin{gathered}
H_{2}^{*}\left(K_{1}, t_{1}\right)-H_{1}^{*}\left(K_{1}, t_{1}\right)=0 \Rightarrow \\
\ln \left[\frac{q_{2}}{q_{1}}\right] e^{-\rho t_{1}}+\left(\frac{q_{2} A_{2}-q_{1} A_{1}}{\rho}\right)\left(e^{-\rho T}-e^{-\rho t_{1}}\right)=0 \Rightarrow \\
t_{1}^{*}=T+\frac{1}{\rho} \ln \left[1-\frac{\rho \ln \left[\frac{q_{2}}{q_{1}}\right]}{\left(q_{2} A_{2}-q_{1} A_{1}\right)}\right] .
\end{gathered}
$$

It follows that there exists an interior solution such that $0<t_{1}^{*}<T$ for a given $T>0$, if and only if

$$
0<\frac{\rho \ln \left[\frac{q_{2}}{q_{1}}\right]}{\left(q_{2} A_{2}-q_{1} A_{1}\right)}<1-e^{-\rho T} .
$$


3. We will check the second order condition whether $t_{1}^{*}$ leads to a maximum or not. For $t_{1}^{*}$ to be a welfare maximizing switching time, sufficient condition is that, at $t_{1}^{*}$,

$$
\frac{\partial H_{2}^{*}\left(K_{1}, t_{1}\right)}{\partial t_{1}}-\frac{\partial H_{1}^{*}\left(K_{1}, t_{1}\right)}{\partial t_{1}}<0 .
$$

Therefore,

$$
\frac{\rho \ln \left[\frac{q_{2}}{q_{1}}\right]}{\left(q_{2} A_{2}-q_{1} A_{1}\right)}>1 .
$$

Note that this violates the condition under which an interior solution exists such that $0<t_{1}^{*}<T$, for a given $T>0$. The date $t_{1}^{*}$ computed in step 3 . is not a maximizer. ${ }^{8}$

4. We finally investigate under which parametric conditions the corner solutions arise. Since $q_{2}>q_{1}$, the condition just above can be broken in two ways.

(i) Technological sclerosis: If $q_{2} A_{2}-q_{1} A_{1} \leq 0$, the interior solution does not exist. Indeed under this condition, we have always $H_{2}^{*}\left(K_{1}, t_{1}\right)-H_{1}^{*}\left(K_{1}, t_{1}\right)>$ 0 for every $0 \leq t_{1} \leq T$ as long as $q_{2}>q_{1}$. This implies that there is no incentive to switch in such a case. If $q_{2} A_{2}-q_{1} A_{1}>0$ but $\frac{\rho \ln \left[\frac{q_{2}}{q_{1}}\right]}{\left(q_{2} A_{2}-q_{1} A_{1}\right)}>$ $1-e^{-\rho T}$, we have still, $H_{2}^{*}\left(K_{1}, t_{1}\right)-H_{1}^{*}\left(K_{1}, t_{1}\right)>0$ for every $0 \leq t_{1}<T$, and $H_{2}^{*}\left(K_{T}, T\right)-H_{1}^{*}\left(K_{T}, T\right) \geq 0$ : Switching is not optimal neither.

(ii) Immediate switching: If $t_{1}^{*}=0$ then $H_{1}^{*}(0) \geq H_{2}^{*}(0)$. Therefore,

$$
\rho \ln \left[\frac{q_{1}}{q_{2}}\right]+\left(1-e^{-\rho T}\right)\left(q_{2} A_{2}-q_{1} A_{1}\right) \geq 0,
$$

or

$$
1-e^{-\rho T} \geq \frac{\rho \ln \left[\frac{q_{2}}{q_{1}}\right]}{\left(q_{2} A_{2}-q_{1} A_{1}\right)} .
$$

It must be noted from the parametric conditions under which we obtain corner solutions that switching to the new technology with a delay is always dominated by either switching immediately or sticking to the old technology alternatives.

\subsection{Economic interpretation}

We now concisely interpret the results above. We summarize them in the following proposition. 
Proposition 1. Assume $q_{2}>q_{1}$ and $A_{1}>A_{2}$.

(i) If $q_{2} A_{2}-q_{1} A_{1} \leq 0$, the economy will not switch to the new technology.

(ii) If $q_{2} A_{2}-q_{1} A_{1}>0$, then:

(v) either $\frac{\rho \ln \left[\frac{q_{2}}{q_{1}}\right]}{\left(1-e^{-\rho T}\right)\left(q_{2} A_{2}-q_{1} A_{1}\right)}<1$, and the economy immediately switches to the new technology,

(vv) or $\frac{\rho \ln \left[\frac{q_{2}}{q_{1}}\right]}{\left(1-e^{-\rho T}\right)\left(q_{2} A_{2}-q_{1} A_{1}\right)}>1$, the economy sticks to the old technology,

(vvv) or $\frac{\rho \ln \left[\frac{q_{2}}{q_{1}}\right]}{\left(1-e^{-\rho T}\right)\left(q_{2} A_{2}-q_{1} A_{1}\right)}=1$, the economy is indifferent between switching or sticking.

These results come from some specific outcomes of the embodiment assumption. In order to understand them easily, note that from our computations in the previous section, optimal consumption follows the path:

$$
C(t)=\alpha e^{\left(q_{1} A_{1}-\rho\right)\left(t-t_{1}\right)}
$$

if $0 \leq t<t_{1}$, and

$$
C(t)=\beta e^{\left(q_{2} A_{2}-\rho\right)\left(t-t_{1}\right)},
$$

for $t_{1}<t \leq T$, with $\alpha$ and $\beta$ two constants such that $\frac{\alpha}{\beta}=\frac{q_{2}}{q_{1}}$. (27) is obtained by combining (13),(15) and (16), and (28) is obtained by combining (21), (23) and (24). Notice the two following characteristics of optimal consumption:

$\left(P_{1}\right)$ : The consumption path is discontinuous at $t=t_{1}$. Since $q_{2}>q_{1}$, we have $\alpha>\beta$, meaning that the level of consumption along the old technology regime is higher than in the new regime.

$\left(P_{2}\right)$ : The growth rate of consumption is higher in the second regime if and only if $q_{2} A_{2}-q_{1} A_{1}>0$, that is if the improvement in the efficiency of the new capital goods is enough to compensate the loss in expertise (ie. $A_{2}<A_{1}$ ) due to the switch.

Let us interpret further property $\left(P_{1}\right)$. The acceleration in the rate of embodied technological change is associated in our framework with a reduction in the relative (shadow) price of capital. In other words, this acceleration is accompanied by a rise in the (shadow) price of consumption goods with respect to the (shadow) price of capital goods. This induces a re-assignment of the resources of the economy towards the capital goods sector, resulting in relatively less consumption and more capital accumulation. So the consumption level should drop from the date of the switch. Since the utility function is logarithmic here, the welfare cost of this drop is $\rho \ln \left(\frac{q_{2}}{q_{1}}\right)$. The 
higher the impatience rate $\rho$, the bigger the welfare cost due to the drop in the consumption level. We shall refer to this cost as the the obsolescence costs inherent to embodiment as it derives from the decrease in the relative (shadow) price of capital.

On the other hand, if the economy switches to the new regime and if the resulting improvement in the efficiency of capital goods is enough to compensate the loss in expertise, the economy will experience a higher growth rate. The growth rate differential is $q_{2} A_{2}-q_{1} A_{1}>0$. As the horizon is finite, the growth rate advantage is exactly: $\left(1-e^{-\rho T}\right)\left(q_{2} A_{2}-q_{1} A_{1}\right)$. As $T$ shrinks, this advantage gets smaller. The trade-off is clear and can be mea-

sured by the ratio cost to advantage of switching, $\Phi=\frac{\rho \ln \left(\frac{q_{2}}{q_{1}}\right)}{\left(1-e^{-\rho T}\right)\left(q_{2} A_{2}-q_{1} A_{1}\right)}$. If $\Phi>1$, the economy has no incentive to switch (case (ii)-(vv) of Proposition 1): The obsolescence costs more than compensate the growth advantage from switching. If in contrast $\Phi<1$, the economy should switch immediately as the growth advantage is the dominating force in such a case.

Notice that in this benchmark model, delaying technology adoption is never optimal, even when the obsolescence costs coming from switching are close to the corresponding growth advantage ( $\Phi$ slightly lower than 1$)$. The rational behind this behavior is the following. ${ }^{9}$ Because of property $\left(P_{1}\right)$, a technology switching implies a downward jump in the level of consumption. Because the utility function is strictly concave, the planner tends to smooth consumption, which means that she typically avoids consumption discontinuities whenever possible. Consumption discontinuities may be optimal if the planner is surprised or if there are some incentives to delay switching. Since the planner has perfect foresight and no incentive to wait in our benchmark model, consumption discontinuities are not allowed after $t=0$. Therefore, delaying adoption is never optimal in the benchmark case. In the next section, we will amend the model in order to generate optimal adoption delays. Before, we examine very briefly the infinite time horizon extension of the benchmark.

\subsection{Infinite Horizon Case}

In this section, we study the infinite horizon extension of our benchmark model, $T=\infty$. One may follow exactly the computation steps detailed in our application to the benchmark model. This is not necessary if one has in mind that the solution paths of the infinite horizon problems are likely to be the limits of the solution paths obtained in the finite horizon case when $T$ tends to infinity. Indeed, the optimization horizon crucially enters step 1 of the method, the so called new technology Pontryagin problem. In particular, the terminal conditions (11) are very important: $\lambda_{2}^{*}(T) \leq$ 
0 and $K^{*}(T) \lambda_{2}^{*}(T)=0$. Since the objective function of our problem is strictly concave in the control variable and since it is discounted, the needed transversality conditions are just the limits of the terminal conditions (11) when $T$ goes to infinity:

$$
\lim _{t \rightarrow \infty} K^{*}(t) \lambda_{2}^{*}(t)=0
$$

with $\lim _{t \rightarrow \infty} \lambda_{2}^{*}(T) \leq 0$. As one can check, this is the unique departure with respect to the benchmark case, in steps 1 and 2.1. In particular, the optimal consumption path follows a similar motion:

$$
C(t)=\alpha^{\prime} e^{\left(q_{1} A_{1}-\rho\right)\left(t-t_{1}\right)},
$$

if $0 \leq t<t_{1}$, and

$$
C(t)=\beta^{\prime} e^{\left(q_{2} A_{2}-\rho\right)\left(t-t_{1}\right)},
$$

for $t_{1}<t$, with $\alpha^{\prime}$ and $\beta^{\prime}$ two positive. Again, one can check that as in the benchmark case, $\frac{\alpha^{\prime}}{\beta^{\prime}}=\frac{q_{2}}{q_{1}}$. Again, we have property $\left(P_{1}\right)$ : An acceleration in embodied technical progress induces a drop in the consumption level. How does the growth rate advantage of the new regime play now? Recall that in the finite horizon case, this advantage is captured by the term (1 $\left.e^{-\rho T}\right)\left(q_{2} A_{2}-q_{1} A_{1}\right)$. In the infinite horizon case, this advantage is simply $q_{2} A_{2}-q_{1} A_{1}$. If $q_{2} A_{2}-q_{1} A_{1} \leq 0$, then there is no incentive to switch. What happens if $q_{2} A_{2}-q_{1} A_{1}>0$ ? Is there any way to generate interior switching time? Our economic interpretations in the last paragraph of section 2.3 suggest a negative answer to this question. Either with a finite or an infinite time horizon, consumption discontinuities are not optimal after $t=0$ under perfect foresight and strictly concave preferences. This diagnosis is confirmed by the method. Indeed, following closely step 2.2 of the method, one ends with the following expression for $H_{2}^{*}\left(K_{1}, t_{1}\right)-H_{1}^{*}\left(K_{1}, t_{1}\right)$ :

$$
H_{2}^{*}\left(K_{1}, t_{1}\right)-H_{1}^{*}\left(K_{1}, t_{1}\right)=\ln \left[\frac{q_{2}}{q_{1}}\right] e^{-\rho t_{1}}+\left(\frac{q_{2} A_{2}-q_{1} A_{1}}{\rho}\right)\left(-e^{-\rho t_{1}}\right) .
$$

For an interior solution to exist, we must have $H_{2}^{*}\left(K_{1}, t_{1}\right)-H_{1}^{*}\left(K_{1}, t_{1}\right)=0$. A quick look at the expression above is sufficient to see that the latter equation (in $t_{1}$ ) is degenerate. We then trivially identify the following cases.

Proposition 2. Assume $q_{2}>q_{1}$ and $A_{1}>A_{2}$.

(i) If $q_{2} A_{2}-q_{1} A_{1} \leq 0$, the economy will not switch to the new technology.

(ii) If $q_{2} A_{2}-q_{1} A_{1}>0$, then:

(w) or $1>\frac{\rho \ln \left[\frac{q_{2}}{q_{1}}\right]}{\left(q_{2} A_{2}-q_{1} A_{1}\right)}$, the economy switches immediately to the new technological regime,

(ww) or $\frac{\rho \ln \left[\frac{q_{2}}{q_{1}}\right]}{\left(q_{2} A_{2}-q_{1} A_{1}\right)}>1$, the economy sticks to the old technology. ${ }^{10}$ 
Hence, independently of the optimization time horizon is infinite, only two decisions might be optimal and both are corner solutions: Either immediate switching when the obsolescence costs are lower than the growth rate advantage of switching (case (ii)-(w)of Proposition 2), or sticking to the old technology in the reverse situation (case (ii)-(ww) of Proposition 2). We next examine a much richer model including learning and incentives to wait.

\section{Optimal technology switching with learn- ing}

We consider the following extension of the benchmark model. The optimization horizon is infinite as in section 2.3 just above. The production function is still AK but the productivity parameter is now time varying to allow for learning effects, exactly as in Greenwood and Jovanovic (2001). Precisely we write: $Y(t)=A(t) K(t)$ with : $A(t)=1$ if $0 \leq t<t_{1}$ and:

$$
A(t)=1-A^{\star} e^{-\theta\left(t-t_{1}\right)},
$$

for $t_{1}<t$, with $A^{\star}=b e^{-\kappa t_{1}}+\alpha_{0}\left(\frac{q_{2}}{q_{1}}\right)$, where $\theta, \kappa, b$ and $\alpha_{0}$ are positive parameters. Contrary to the benchmark case, the loss in expertise following a technology switch is not permanent. The economy is supposed to learn more about the new technology time after time, and to asymptotically eliminate this expertise gap. The learning speed is measured by the parameter $\theta$. The amount to be learnt at the date of the switch is given by $A^{\star}$. Note that this amount increases as the technological acceleration $\frac{q_{2}}{q_{1}}$ gets sharper. Notice also that the learning costs are decreasing with respect to the switching time (since $b$ and $\kappa$ are positive): The later the switching date, the lower the amount to be learnt. ${ }^{11}$

This specification is crucial in generating interior switching dates as we will see later. In our framework, such a behavior is not possible if the amount to be learnt is independent of the switching date. If the amount to be learnt is increasing in this date (for example if $b$ is positive and $\kappa$ is negative), delaying adoption is also sub-optimal since we have a further mechanism to add to those identified in the benchmark case against waiting. Our modelling (both $b$ and $\kappa$ positive) introduces an incentive to wait. It is consistent with the typical network externalities or late mover advantage stories often invoked to build up diffusion curves (again see Greenwood and Jovanovic, 2001, for an excellent survey of the literature regarding this aspect). How do the learning mechanism and the waiting incentives affect the solution to the optimal adoption problem seen in section 2.3? What could be an optimal adoption delay with these new specifications? 
Before solving this problem using two stage optimal control, a very important observation should be made. The law of motion of capital writes as follows:

$$
\dot{K}(t)=q_{1}[K(t)-c(t)]
$$

if $0 \leq t<t_{1}$, and

$$
\dot{K}(t)=q_{2}\left[\left(1-A^{\star} e^{-\theta\left(t-t_{1}\right)}\right) K(t)-c(t)\right],
$$

for $t>t_{1}$. With respect to the law of motion of capital (1)-(2) in the benchmark case, the switching date $t_{1}$ does enter the expression of $\dot{K}(t)$ for $t>t_{1}$. Naturally this should change the optimality condition with respect to $t_{1}$, and the steps 2.2 and 3 of the method, as derived from to Tomiyama (1985), should be amended. This issue has been indeed tackled by Tomiyama and Rossana (1989), and we will illustrate their approach hereafter.

\subsection{Solving the model}

1. The Pontryagin conditions corresponding to the new technology problem are as before $(9),(10)$ with the transversality conditions $\lim _{t \rightarrow \infty} K^{*}(t) \lambda_{2}^{*}(t)=$ 0 and $\lim _{t \rightarrow \infty} \lambda_{2}^{*}(T) \leq 0$. The corresponding Hamiltonian is

$H_{2}\left(K, c, t, \lambda_{2}\right)=-\ln (C(t)) e^{-\rho t}+\lambda_{2}\left(q_{2}\left(1-\left(b e^{-\kappa t_{1}}+\alpha_{0} \frac{q_{2}}{q_{1}}\right) e^{-\theta\left(t-t_{1}\right)}\right) K(t)-q_{2} C(t)\right)$.

Integrating the Pontryagin conditions, one finds after some trivial but heavy algebra that:

$$
\begin{aligned}
& K(t)=e^{q_{2}\left(t+\frac{e^{-\theta\left(t-t_{1}\right)}\left(b q_{1} e^{-t_{1} \kappa}+q_{2} \alpha_{0}\right)}{q_{1} \theta}\right)}\left(\frac{q_{2} a_{0}}{\rho}\left(e^{-\rho t}-e^{-\rho t_{1}}\right)+e^{-q_{2}\left(t_{1}+\frac{b e^{-t_{1} \kappa}+\alpha_{0}}{\theta}\right)} K_{1}\right), \\
& C(t)=a_{0} e^{\left(q_{2}-\rho\right) t+\frac{e^{-\theta\left(t-t_{1}\right)} q_{2}\left(b e^{-t} \kappa_{1} q_{1}+q_{2} \alpha_{0}\right)}{q_{1} \theta}}, \\
& \lambda_{2}(t)=-\frac{1}{q_{2} a_{0}} e^{-q_{2} t-\frac{e^{-\theta\left(t-t_{1}\right)} q_{2}\left(b e^{-t_{1} \kappa} q_{1}+q_{2} \alpha_{0}\right)}{q_{1} \theta}} .
\end{aligned}
$$

Finally the transversality conditions give:

$$
a_{0}=\frac{K_{1} \rho}{q_{2}} e^{\left(\rho-q_{2}\right) t_{1}-\frac{q_{2}\left(b e^{-t_{1} \kappa} q_{1}+q_{2} \alpha_{0}\right)}{q_{1} \theta}} .
$$

Now come the new things with respect to the benchmark case application. Using the expressions above for optimal consumption, capital accumulation 
and the co-state variable, one can again express the optimal value of the objective function as a function $U_{2}\left(K_{1}, t_{1}\right)$. It is then possible to check that:

$$
\begin{gathered}
\frac{\partial U_{2}^{\star}\left(K_{1}, t_{1}\right)}{\partial K_{1}}=-\lambda_{2}\left(t_{1}\right), \\
\frac{\partial U_{2}^{\star}\left(K_{1}, t_{1}\right)}{\partial t_{1}} \equiv H_{2}^{*}\left(K_{1}, \lambda_{2}^{*}\left(t_{1}\right), C^{*}\left(t_{1}\right), t_{1}\right)-\int_{t_{1}}^{\infty} \frac{\partial H_{2}^{*}}{\partial t_{1}} d t .
\end{gathered}
$$

With respect to the benchmark case, a new term appears in the expression of $\frac{\partial U_{2}^{\star}\left(K_{1}, t_{1}\right)}{\partial t_{1}}$, namely $\int_{t_{1}}^{\infty} \frac{\partial H_{2}^{*}}{\partial t_{1}} d t$. This term captures the effects of a change in the switching date on the Hamiltonian value, and so on the optimal welfare performance of the second technology regime. It is present here because the switching date value affects explicitly the pace of capital accumulation through the learning costs. This new term will obviously appear again in the matching conditions in step 2.2.

2.1. Since $H_{1}\left(K, c, t, \lambda_{1}\right)=-\ln (C(t)) e^{-\rho t}+\lambda_{1} q_{1}(K(t)-C(t))$, this step is identical to step 2.1 in section 2.2. So equations (20), (21) and (22) still hold with $A_{1}=1$. In particular, we get:

$$
C(t)=\bar{a}_{0} e^{\left(q_{1}-\rho\right) t}
$$

$\bar{a}_{0}$ the integration constant.

2.2. The optimality condition of the problem with respect to $K_{1}$ does not change, equation (4) still applies. However, since $t_{1}$ appears directly in the accumulation law of capital along the second technological regime, a new optimality condition arises with respect to $t_{1}$ :

$$
\frac{\partial U_{2}^{*}\left(K_{1}, t_{1}\right)}{\partial t_{1}}=H_{1}^{*}\left(K_{1}, t_{1}\right)+\int_{0}^{t_{1}} \frac{\partial H_{1}^{*}}{\partial t_{1}} d t
$$

Using the results of step 1, one can check that this condition can be rewritten as

$$
H_{2}^{*}\left(K_{1}, t_{1}^{*}\right)-H_{1}^{*}\left(K_{1}, t_{1}^{*}\right)=\int_{0}^{t_{1}^{*}}\left(\frac{\partial H_{1}^{*}}{\partial t_{1}}\right) d t+\int_{t_{1}^{*}}^{\infty}\left(\frac{\partial H_{2}^{*}}{\partial t_{1}}\right) d t
$$

while, as in the benchmark case, equation (6), the optimality condition with respect to $K_{1}$ may be rewritten as a continuity requirement at $t=t_{1}$ of the co-state variable. From this continuity condition, $\lambda_{1}\left(t_{1}\right)=\lambda_{2}\left(t_{1}\right)=\frac{-e^{-\rho t_{1}}}{\rho K_{1}}$, 
one can compute the integration constant $\bar{a}_{0}$ as: $\bar{a}_{0}=\frac{\rho K_{1}}{q_{1}} e^{\left(\rho-q_{1}\right) t_{1}}$, which allows to derive the optimal paths of consumption, capital accumulation and the co-state variable in terms of $t_{1}$. In particular, $K_{1}=K\left(t_{1}\right)=K_{0} e^{\left(q_{1}-\rho\right) t_{1}}$. Substituting in the (interior) optimality condition with respect to $t_{1}$, namely in equation (34), one gets after some trivial but cumbersome algebraic operations that:

$$
t_{1}^{*}=-\frac{1}{\kappa} \ln \left[\frac{q_{1}(\theta+\rho)\left(q_{2}-q_{1}-\rho \ln \left[\frac{q_{2}}{q_{1}}\right]\right)-q_{2}^{2} \alpha_{0} \rho}{b q_{1} q_{2}(\kappa+\rho)}\right]
$$

One can easily check that optimal $t_{1}^{*} \in(0, \infty)$ if and only if

$$
\frac{q_{2}^{2} \alpha_{0} \rho}{q_{1}(\theta+\rho)}<\left(q_{2}-q_{1}-\rho \ln \left[\frac{q_{2}}{q_{1}}\right]\right)<\frac{q_{2}^{2} \alpha_{0} \rho}{q_{1}(\theta+\rho)}+\frac{b q_{2}(\kappa+\rho)}{(\theta+\rho)} .
$$

3. The sufficient condition for $t_{1}^{*} \in(0, \infty)$ to be welfare maximizing is:

$$
\frac{\partial H_{2}^{*}\left(K_{1}, t_{1}\right)}{\partial t_{1}}-\frac{\partial H_{1}^{*}\left(K_{1}, t_{1}\right)}{\partial t_{1}}<\frac{\partial}{\partial t_{1}}\left(\int_{0}^{t_{1}}\left(\frac{\partial H_{1}^{*}}{\partial t_{1}}\right) d t+\int_{t_{1}^{*}}^{\infty}\left(\frac{\partial H_{2}}{\partial t_{1}}\right) d t\right)
$$

which can be recast as

$$
\left(q_{2}-q_{1}-\rho \ln \left[\frac{q_{2}}{q_{1}}\right]\right)<\frac{q_{2}^{2} \alpha_{0} \rho}{q_{1}(\theta+\rho)}+\frac{b q_{2}(\kappa+\rho)^{2} e^{-\kappa t_{1}}}{\rho(\theta+\rho)} .
$$

It can be easily checked that at $t_{1}^{*} \in(0, \infty)$, given by $(35)$, the sufficient condition reduces to

$$
\left(q_{2}-q_{1}-\rho \ln \left[\frac{q_{2}}{q_{1}}\right]\right)>\frac{q_{2}^{2} \alpha_{0} \rho}{q_{1}(\theta+\rho)},
$$

which is always satisfied.

4. The necessary conditions for the degenerate cases where either the optimal switching appears immediately or never switching occurs can be found very easily.

(i) Technological sclerosis: This case occurs if $H_{1}^{*}\left(K_{1}, t_{1}^{*}\right)-H_{2}^{*}\left(K_{1}, t_{1}^{*}\right) \leq$ $\int_{0}^{t_{1}^{*}}\left(\frac{\partial H_{1}^{*}}{\partial t_{1}}\right) d t+\int_{t_{1}^{*}}^{\infty}\left(\frac{\partial H_{2}^{*}}{\partial t_{1}}\right) d t$ if $t_{1}^{*} \rightarrow \infty$. This happens if and only if the following condition holds

$$
\left(q_{2}-q_{1}-\rho \ln \left[\frac{q_{2}}{q_{1}}\right]\right) \leq \frac{q_{2}^{2} \alpha_{0} \rho}{q_{1}(\theta+\rho)}
$$


(ii) Immediate switching: The economy switches immediately if $H_{1}^{*}\left(K_{1}, t_{1}^{*}\right)-$ $H_{2}^{*}\left(K_{1}, t_{1}^{*}\right) \geq \int_{0}^{t_{1}^{*}}\left(\frac{\partial H_{1}^{*}}{\partial t_{1}}\right) d t+\int_{t_{1}^{*}}^{\infty}\left(\frac{\partial H_{2}^{*}}{\partial t_{1}}\right) d t$ when $t_{1}^{*}=0$. The corresponding parametric condition is

$$
\left(q_{2}-q_{1}-\rho \ln \left[\frac{q_{2}}{q_{1}}\right]\right) \geq \frac{q_{2}^{2} \alpha_{0} \rho}{q_{1}(\theta+\rho)}+\frac{b q_{2}(\kappa+\rho)}{(\theta+\rho)} .
$$

\subsection{Economic interpretations and comparative statics}

Denote by $\Delta=q_{2}-q_{1}-\rho \ln \left(\frac{q_{2}}{q_{1}}\right)-\frac{q_{2}^{2} \alpha_{0} \rho}{q_{1}(\theta+\rho)}$. The results of the previous section can be summarized by the following proposition.

Proposition 3. Assume $q_{2}>q_{1}$.

(i) If $0<\Delta<\frac{b q_{2}(\kappa+\rho)}{(\theta+\rho)}$, then adoption is delayed, the optimal switching date $t_{1}^{\star}$ being given by (35).

(ii) If $\Delta \leq 0$, the economy should stick to the old technology.

(iii) If $\Delta \geq \frac{b q_{2}(\kappa+\rho)}{(\theta+\rho)}$, the economy should switch immediately to the new technology.

In order to improve the readability of our mathematical results and their economic discussion, we state the optimization outcomes in the special case where the amount to be learnt is constant, ie. $b=0$.

Corollary 1. Under $q_{2}>q_{1}$, if $b=0$, then either technological sclerosis or immediate switching occurs:

$$
\begin{aligned}
& \left(q_{2}-q_{1}-\rho \ln \left[\frac{q_{2}}{q_{1}}\right]\right)<\frac{q_{2}^{2} \alpha_{0} \rho}{q_{1}(\theta+\rho)} \Longleftrightarrow \text { technological sclerosis, } \\
& \left(q_{2}-q_{1}-\rho \ln \left[\frac{q_{2}}{q_{1}}\right]\right)>\frac{q_{2}^{2} \alpha_{0} \rho}{q_{1}(\theta+\rho)} \Longleftrightarrow \text { immediate switching, } \\
& \left(q_{2}-q_{1}-\rho \ln \left[\frac{q_{2}}{q_{1}}\right]\right)=\frac{q_{2}^{2} \alpha_{0} \rho}{q_{1}(\theta+\rho)} \Longleftrightarrow \text { indifferent between switching and sticking. }
\end{aligned}
$$

As in the benchmark case, we have again to deal with the obsolescence costs and growth advantages of adopting a new technology with a higher level of embodied technological progress. Obsolescence costs are still measured by $\rho \ln \left[\frac{q_{2}}{q_{1}}\right]$. Indeed, the optimal consumption path is given by:

$$
C(t)=\frac{\rho K_{1}}{q_{1}} e^{\left(q_{1}-\rho\right)\left(t-t_{1}\right)}, \text { if } 0 \leq t<t_{1}
$$


and

$$
C(t)=\frac{\rho K_{1}}{q_{2}} e^{\left(q_{2}-\rho\right)\left(t-t_{1}\right)-\frac{\alpha_{0} q_{2}^{2}+q_{1} q_{2} b e^{-\kappa t_{1}}}{q_{1} \theta}\left(1-e^{-\theta\left(t-t_{1}\right)}\right)}, \text { if } t_{1}<t .
$$

Taking the one-sided limits of the consumption path at $t_{1}$ is enough to see that the obsolescence costs, at the basis of the drop in the level of consumption, can be measured exactly as in the benchmark case by $\rho \ln \left[\frac{q_{2}}{q_{1}}\right]$. The growth advantage of switching is now measured by $q_{2}-q_{1}$ since both regimes imply the same long term marginal productivity of capital, equal to 1. Nonetheless, for this to hold, the economy should "learn" once it switches, and this is costly. Now recall the learning curve structure: $A(t)=$ $1-A^{\star} e^{-\theta\left(t-t_{1}\right)}$, for $t_{1}<t$, with $A^{\star}=b e^{-\kappa t_{1}}+\alpha_{0}\left(\frac{q_{2}}{q_{1}}\right)$. The amount to be learnt, $A^{\star}$, consists of two different terms. The first one related to $b e^{-\kappa t_{1}}$ does depend on the value of $t_{1}$ and represents a kind of value option of waiting. By choosing $t_{1}$, the economy controls indeed this component of the learning process. The second term related to $\alpha_{0}\left(\frac{q_{2}}{q_{1}}\right)$ does not depend on $t_{1}$ and captures the fixed learning costs. The economy has no control on this term: It exclusively depends on the intrinsic characteristics of the two considered technological menus, and, as such, this term is not "manipulable" by the potential adopters. The indicator $\Delta$ constructed above is consequently the growth advantage of switching net of the obsolescence costs and the fixed switching costs. Obviously, if $\Delta \leq 0$, the economy has no incentive to switch (case (ii) of Proposition 3).

When $\Delta>0$, the economy will switch. If $\Delta$ is big enough to "compensate" the option of waiting, namely the term $\frac{b q_{2}(\kappa+\rho)}{(\theta+\rho)}$, then there is no reason to wait, and the economy will switch immediately (case (iii) of Proposition 3). Otherwise, the economy will wait, and a strictly positive adoption delay will arise (case (i) of Proposition 3). If the amount to be learnt is constant (Corollary 1), the value option of waiting is nil, implying that an interior switching time is never optimal. In such a case, the cost of switching technologies is simply increased with respect to the benchmark model, learning costs add to the obsolescence costs: There is no substantial departure from the benchmark model, and the economy either "sticks" or immediately switches.

What are the determinants of the optimal adoption delay if it arises? The next proposition summarizes the results regarding this aspect.

Proposition 4. Assume that $0<\Delta<\frac{b q_{2}(\kappa+\rho)}{(\theta+\rho)}$, so that switching is delayed, $t_{1}^{\star}$ given by equation (35). Then $\frac{\partial t_{1}^{*}}{\partial \theta}<0$ and $\frac{\partial t_{1}^{*}}{\partial \alpha_{0}}>0$. In contrast, the sign of $\frac{\partial t_{1}^{*}}{\partial \rho}$ is ambiguous. A sufficient condition for $\frac{\partial t_{1}^{*}}{\partial \rho}$ to be positive is $\rho<\frac{1-\theta}{2}$.

The properties stated in the proposition come from the expressions of the 
derivatives. Indeed:

$$
\frac{\partial t_{1}^{*}}{\partial \theta}=-\frac{1}{\kappa}\left(\frac{q_{1}\left(q_{2}-q_{1}-\rho \ln \left[\frac{q_{2}}{q_{1}}\right]\right)}{q_{1}(\theta+\rho)\left(q_{2}-q_{1}-\rho \ln \left[\frac{q_{2}}{q_{1}}\right]\right)-q_{2}^{2} \alpha_{0} \rho}\right)<0,
$$

as both the denominator and the numerator inside the brackets are positive (due to the condition of interior switching existence). This is far from surprising. A higher value for $\theta$ means a faster learning process, which rises the incentives to switch. On the other hand, one gets immediately

$$
\frac{\partial t_{1}^{*}}{\partial \alpha_{0}}=-\frac{1}{\kappa}\left(\frac{-q_{2}^{2} \rho}{q_{1}(\theta+\rho)\left(q_{2}-q_{1}-\rho \ln \left[\frac{q_{2}}{q_{1}}\right]\right)-q_{2}^{2} \alpha_{0} \rho}\right)>0 .
$$

$\alpha_{0}$ measures the sensitivity of the fixed learning costs to the magnitude of the technological acceleration as captured by the ratio $\frac{q_{2}}{q_{1}}$. Thus an increase in $\alpha_{0}$ rises the learning fixed costs and discourages switching. In contrast, the behavior of adoption timing with respect to time discounting is again ambiguous. In effect:

$$
\frac{\partial t_{1}^{*}}{\partial \rho}=-\frac{1}{\kappa}\left(\frac{q_{1}\left(q_{2}-q_{1}-\rho \ln \left[\frac{q_{2}}{q_{1}}\right]\right)-q_{2}^{2} \alpha_{0} \rho-q_{1}(\theta+\rho) \ln \left[\frac{q_{2}}{q_{1}}\right]}{q_{1}(\theta+\rho)\left(q_{2}-q_{1}-\rho \ln \left[\frac{q_{2}}{q_{1}}\right]\right)-q_{2}^{2} \alpha_{0} \rho}\right) .
$$

The denominator inside the brackets is positive due to the condition ensuring the existence of an interior switching date. The sign of the numerator is ambiguous. A rise in $\rho$ increases the obsolescence costs, $\rho \ln \left[\frac{q_{2}}{q_{1}}\right]$, which tends to delay adoption. However a more "impatient" economy would also accelerate adoption since the sooner the adoption, the sooner the economy will start to learn and the quicker it will gain expertise on the new technology. This aspect is captured by the term $\theta+\rho$ in (35) for example. Therefore, the adoption delay is a very complex function of the impatience rate. A sufficient condition for $\frac{\partial t_{1}^{*}}{\partial \rho}>0$ is that $\rho<\frac{1-\theta}{2}$. If the learning speed is very low (that is $\theta$ tends to zero), this condition is checked by far for reasonable values of the impatience rate. In such a case, the obsolescence costs effects dominate the learning incentive, and the adoption delay is increased.

\section{Conclusion}

In this paper, we have applied two stage optimal control techniques to solve explicitly optimal adoption problems under embodiment. In contrast to 
most of the contributions in the field of technology adoption, our results are analytical. We have first solved a benchmark model without learning behavior. The main property of our model is the drop in the level of consumption at the switching date, coming from the fall in the relative price of capital (obsolescence). If the growth advantage deriving from switching to a new economy with a higher degree of embodiment is bigger than the resulting obsolescence costs, the economy switches immediately; otherwise, it sticks to the initial technical menu. Delaying adoption, waiting is never optimal in such a case. In a second step, we have introduced a learning process accompanying the switch to the new technology when it occurs. Along the considered learning curves, the amount to be learnt is composed of involves some fixed costs and incentives to wait as well. We have shown that the adoption decision depends on the growth advantage of switching net of the obsolescence costs and the learning fixed costs. The economy will switch if and only if this indicator is positive. If it is big enough to "compensate" the option of waiting, then there is no reason to wait, and the economy will switch immediately. Otherwise, the economy will wait, and a strictly positive adoption delay will arise.

Further extensions may consider at least two directions. First, the learning process could be much more finely specified. Second, instead of solving central planner problems, decentralized equilibria could be considered. In such a case, firm heterogeneity should imply differences in adoption timing across sectors and firms, giving rise to diffusion curves. Both issues are in our research agenda. 


\section{Notes}

${ }^{1}$ The Lisbon European Union meeting two years ago has indeed stressed the need to implement a rational pace of adoption of the information technologies in order to take advantage of all its opportunities.

${ }^{2}$ As an example, think about the massive implementation of Minitel, a kind of "local" Internet, in France in the 80s. The surge of Internet makes this huge and too early investment completely useless.

${ }^{3}$ As a matter of fact, the production function is also linear in Parente (1994).

${ }^{4}$ Indeed, it is not difficult to show that this two sector model is the reduced form of the Solow vintage capital model (1960), as pointed out by Boucekkine, del Río and Licandro (in press).

${ }^{5}$ We take the same conventions as Tomiyama (1985).

${ }^{6}$ Since time is continuous, the corner solution $t_{1}^{*}=T$ also features technological sclerosis.

${ }^{7}$ We omit the ${ }^{*}$ for ease of the notation

${ }^{8} \mathrm{It}$ is indeed a minimizer.

${ }^{9}$ We warmly thank an anonymous referee for pointing out this crucial aspect of the problem, which decisively helps us to identify and correct an algebraic mistake in the first version of this paper, and to improve the economic discussion of the results.

${ }^{10}$ In the equality case: $\frac{\rho \ln \left[\frac{q_{2}}{q_{1}}\right]}{\left(q_{2} A_{2}-q_{1} A_{1}\right)}=1$, the economy is indifferent between switching and sticking. We abstract away from this case hereafter.

${ }^{11}$ The adopted additive form for the amount to be learnt is only for convenience, in order to ease the interpretation of the mathematical results.

\section{$5 \quad$ References}

Boucekkine, R., F. del Río \& O. Licandro (in press) Embodied technological change, learning by doing and the productivity slowdown. Scandinavian Journal of Economics. 
Galor, O. \& O. Moav (2000) Ability-biased technological transition, wage inequality, and economic growth. Quarterly Journal of Economics 115, 469-497.

Galor, O. \& D. Tsiddon (1997) Technological progress, mobility, and economic growth. American Economic Review 87, 363-382.

Greenwood, J., Z. Hercowitz \& P. Krusell (1997) Long-run implications of investment-specific technological change. American Economic Review 87, 342-362.

Greenwood, J. \& M. Yorokoglu (1997) 1974. Carnegie-Rochester Series on Public Policies 46, 49-95.

Greenwood, J. \& B. Jovanovic (2001) Accounting for Growth. In E.Dean, M. Harper and C. Hulten (eds), New Directions in Productivity Analysis, NBER Studies in Income and Wealth Volume 63. Chicago: Chicago University Press.

Iacompetta, M. (2001) Dissemination of technology in market and planned economies. Mimeo, New York University.

Jovanovic, B. \& Y. Nyarko (1996) Learning by doing and the choice of technology. Econometrica 64, 1299-1310.

Jovanovic, B. (1997) Learning and Growth. In D. Kreps and K. Wallis (eds.), Advances in economics, Vol. 2, pp. 318-339. London: Cambridge University Press.

Krusell, P. (1998) Investment-specific R\&D and the decline in the relative price of capital. Journal of Economic Growth 3, 131-141.

Makris, M. (2001) Necessary conditions for infinite horizon discounted twostage optimal control problems. Journal of Economic Dynamics and Control 25, 1935-1950.

Parente, S. (1994) Technology adoption, learning by doing, and economic growth. Journal of Economic Theory 63, 346-369.

Saglam, C. (2002) Optimal sequence of technology adoptions with finite horizon via multi-stage optimal control. Mimeo, IRES-Université catholique de Louvain.

Tomiyama, K. (1985) Two-stage optimal control problems and optimality conditions. Journal of Economic Dynamics and Control 9, 317-337. 
Tomiyama K. \& R. Rossana (1989) Two-stage optimal control problems with an explicit switch point dependence: Optimality criteria and an example of delivery lags and investment. Journal of Economic Dynamics and Control $13,319-337$.

Solow, R. (1960) Investment and technological progress. In K. Arrow, S. Karlin and P. Suppes (eds.), Mathematical Methods in the Social Sciences 1959, pp 89-104. Stanford: Stanford University Press. 